\title{
PATHOLOGY AND PUBLIC HEALTH SIGNIFICANCE OF SALMONELLA
}

\author{
Muhammad Younus ${ }^{* 1}$, Muhammad Asif Idrees ${ }^{1}$, Qamur-un-Nisa², Qaiser Akram and Waqas Ahmad ${ }^{3}$ \\ 'Department of Pathobiology, University College of Veterinary and Animal Sciences, Narowal, 5160o, Pakistan \\ ${ }^{2}$ Department of Pathology, University of Veterinary and Animal Sciences, Lahore, Pakistan \\ 3Department of Clinical Sciences, University College of Veterinary and Animal Sciences, Narowal, 5160o, Pakistan \\ ${ }^{*}$ Corresponding author: younusrana@uvas.edu.pk
}

\section{INTRODUCTION}

Genus Salmonella comprises of bacteria that are gram negative, rod shaped and belong to the family Enterobacteriaceae (Su and Chiu 2007; Arshad et al. 2008).) Organisms of this genus are non-spore forming, intracellular, ubiquitous, catalase positive, oxidase negative, and facultative anaerobes (Jantsch et al. 2011; Cox and Pavic 2014).) This genus has two species i.e. Salmonella (S) enterica and S. bongori (Park et al. 2017). Six subspecies also emerged out from $S$. enterica that include over 2600 serotypes or serovars (IssenhuthJeanjean et al. 2014) which are distinguishable by certain serological and biochemical tests (Fig. 1).

These subspecies include, enterica, salamae, arizonae, diarizonae, indica and houtenae (Vohra et al. 2018). Approximately $99 \%$ of Salmonella infections result from these species (Tekintaş et al. 2018). The most common serovars reported globally are S. typhimurium and $S$. enteritidis, both are commonly found in human and animal cases (Archambault and Petrov 2006; Hendriksen et al. 2011; Manning et al. 2015).

Salmonella infection may manifest either as a self-limiting diarrhea/gastroenteritis, which is related to nontyphoidal Salmonella (NTS) or as typhoidal fever that involves septicemia and chronic diarrhea, and can be life threatening (Barrow and Methner 2013; Cox and Pavic 2014). The first form (NTS) is usually linked with inflammatory diarrhea, vomiting, nausea and abdominal pain (Smith et al. 2016). The severity of infection, like many other infectious diseases, depends upon host immune status, genetic makeup, the specie involved, serovar and dose of inoculation of the pathogen.

\section{Public Health Significance}

Public health issues and the capability for foodborne zoonotic spread have made Salmonella the focus of various international, national, and regional surveillance platforms (Pal et al. 2020). Salmonellosis is a major and economically important public health issue. Globally, an estimation indicates 33 million cases, and 0.5 million deaths associated with typhoid fever (Sandala et al. 2020), while NTS cause 93 million illnesses with 0.155 million deaths each year (Majowicz et al. 2010).

Salmonellosis lies among the most abundant food borne zoonotic diseases, and the disease can be spread to an extensive variety of hosts, including humans, animals and birds (Kaufmann et al. 2001; Jafari et al. 2007; Younus et al. 2011). It can also infect all species of animals including livestock, poultry, pigs, reptiles, amphibians, dogs and cats (Stear 2005). Children under 5 years of age (Su and Chiu 2007), elders and immune-compromised patients are most susceptible. Symptoms of the disease begin to appear 6-7 hours after infection with Salmonella and illness lasts for 2-7 days (Eikmeier et al. 2018). Intake of contaminated food related to animal origin, including meat, unpasteurized milk, raw or under cooked eggs or egg products, contaminated vegetables and fruit generally cause infections in humans (Lambertini et al. 2016). However, other food products, like contaminated green vegetables, can also cause transmission of disease (Cocciolo et al. 2020; Nguyen et al. 2021).

Consumption of poultry products, including eggs and meat, remains one of the leading causes of food borne infections of Salmonella in human population. Salmonella may enter poultry flocks through vertical and horizontal transmissions. The horizontal methods include direct transmission between flocks, contaminated feeds, and biological vectors like birds, insects, and rodents (Cocciolo et al. 2020), ultimately affecting human population. Epidemiological studies show that poultry flocks are infected directly by the environment of poultry farm (Dagnew et al. 2020).

According to US Food and Drug Administration, 2 to 4 million cases of Salmonellosis in humans occur every year only in US (Gerner-Smidt et al. 2006; Hassan et al. 2018). Salmonella causes wide range of diseases with enteric and typhoid fever, food poisoning, diarrhea and gastroenteritis. Many serotypes of Salmonella do not have host specificity and cause disease in animals and humans. Salmonella has the capability to modify according to the climate and it can develop resistance against routine elimination practices of sanitation, chemical treatments and antibacterial drugs. S. enterica serovar Enteritidis is the most common serotype of Salmonella isolated from cases of food borne gastroenteritis throughout the world (Dantas et al. 2018; Das et al. 2019). S. enteritidis is responsible for outbreaks of human food borne salmonellosis and is generally associated with the consumption of poultry products. Inactivated $S$. enteritidis cell vaccine is one of the available methods to control $S$. enteritidis in breeders and laying hens, however, results in terms of efficacy vary (Crouch et al. 2020). Salmonella is an important zoonotic pathogen and its prevalence in the chicken meat and eggs poses a 
continuous challenge to public health. Salmonella enteritidis has been the major cause of the food borne Salmonellosis pandemic in humans over the last 20 years (Raspoet et al. 2011), during which contaminated chicken eggs were the most important vehicle of the infection. Human Salmonellosis infections are usually acquired via the food chain as a result of the ability of Salmonella serovars to colonize and persist within the gastrointestinal tract of their hosts.

\section{Antimicrobial Resistance}

Antibiotics have consistently been viewed as one of the great revelations of the $20^{\text {th }}$ century. The expansion in the use of antibiotics in emergency clinics, networks and the climate are increasing the antimicrobial resistance. The misuse of microorganisms has resulted in the massive economical and financial losses, and enhanced the overall burden of diseases. Antimicrobial resistance of pathogenic microorganisms is a test related with high morbidity and mortality (Johansson et al. 2021).

Antibiotics may be needed in high-risk groups, such as young children, the aged persons, and those with compromised immunity. With respect to the drugs, ampicillin, chloramphenicol, and trimethoprimsulfamethoxazole can be utilized for the treatment of Salmonellosis. However, resistance to these drugs has increased significantly in recent years. Fluoroquinolones have been recommended for the treatment of Salmonella infections for adults, while third generation cephalosporin are the drugs of choice to treat very young patients or when fluoroquinolone resistance is present (Muhammad et al. 2015).

Resistance against the most common antimicrobials used in humans and animal production systems is a major concern in Nigeria, where Multi Drug Resistant (MDR) Salmonella strains are among the most common causes of bacteremia in children (Bogomazova et al. 2020). Salmonella serotypes with reduced sensitivity to fluoroquinolones in humans have been documented (Eguale et al. 2017). A previous study showed that the observed fingerprints had the same RAPD patterns, suggesting that grocers may have been infected from animal sources because the samples were taken from drug sites (Smith et al. 2011).

A major public health concern around the globe is the rapid emergence of antibiotic resistance in all types of bacterial species, especially in Salmonella. Use of antibiotics, even for very necessary purposes, is curbed when this resistance mechanism is developed. In addition to this, the transfer of this resistance potential to next generation makes this situation worse. The unjustified and unchecked use of antibiotics in feed animals favors the development of antibiotic resistance mechanism (Younus et al. 2009). A transferable plasmid is proven to be a source of resistance in many genetic analyses. Recent studies have shown that some serotype-specific virulence plasmids, through recombination with resistance plasmids, form hybrid plasmids or acquire gene cassettes consisting of different resistance genes. Such evolutionary events give virulent strain the advantage of surviving in an adverse pharmacological environment. With respect to serious consequences of drug-resistant Salmonella species, more targeted use of antibiotics in human medicine and the animal industry is needed (Lima et al. 2019). Continuous monitoring of antibiotic resistance and the use of antimicrobial agents in food animals is also essential.

Resistance of Salmonella to Different Classes of Antimicrobial Agents

S. enterica carries antimicrobial resistance genes against some drugs. For example, out of forty tetracycline resistant genes, five have been reported in Salmonella isolates. These include TetA (A, B, C, D and G). An efflux protein, comprising twelve trans-membrane parts, is being encoded by these genes that carries doxycycline, tetracycline, chlortetracycline and oxytetracycline. Minocycline can also be distributed by TetA(B). The five TetA genes are linked with different components, like Tn1721, Tnıo and Salmonella Genomic Island 1 or 2. Tnıo is prevalent among various Salmonella serovars. TetA (G) gene has solely been detected as an element of SGI1 or 2allied with multi resistance gene clusters. In certain conditions, the same isolate was found in more than one TetA gene (Michael et al. 2005). Another group of $\beta$ lactam antibiotics includes penicillins and cephalosporins. $\beta$-lactamase enzymes deactivate the antibiotics, leading to the drug resistance. The $\beta$ lactamases form a distinct cluster of enzymes coded by significant repertory of genes (Michael et al. 2005).

\section{Species of Zoonotic Importance}

One of the major serotypes of S. typhimurium contaminates numerous animal species around the world, including livestock and poultry (Rao et al. 2020). The sort and seriousness of S. typhimurium disease differ through different species. Salmonella disease in calves prompts fever and looseness of the bowels, with high mortality if no anti-toxin treatment is given (Birhanu et al. 2018). S. typhimurium contamination in day-old chicks additionally prompts enteric and other fundamental illnesses, with a high death rate (Nascimento et al. 2019), while disease in more seasoned chicks prompts asymptomatic colonization of dung with relentless discharge of living organisms in defecation (Deng et al. 2020).

S. enterica subspecies enteric serotype typhimurium (S. typhimurium) is perceived as a human microorganism and represents a sanitation hazard around the world. It can taint a wide range of hosts, including poultry, pigs, sheep and steers. S. typhimurium is one of the five important serotypes from food delivering creatures and is a main source of food borne contaminations in various countries (Rao et al. 2020).

S. enterica have two serovars viz S. typhi and S. paratyphi, and these two serovars are capable of producing enteric fever in affected individuals (Monte et al. 2019). Both 
serovars of S. enterica are pathogenic for humans and these are transmitted through oral and fecal routes associated with consumption of unhygienic water and foodstuff. Previous published data reveals many difficulties to observe the burden of this zoonotic disease in different locations, areas and countries, but all have high rate of death due to typhoid caused by these serovars. These Salmonella serovars are present in many countries, causing typhoid fever in Asia and Africa. Reports from some countries also show that its incidence has decreased in these countries.

Salmonella typhi is causing human infections in developing states (Wong et al. 2016). Primary source of transmission is through consuming unhygienic and contaminated food items and drinking of water. On the other hand, domestic birds, such as pigeons, can transmit Salmonella typhi infection to humans (KaczorekŁukowska et al. 2020). In a live bird market of Lahore Pakistan, domestic fowls and many fancy birds are sold and the contamination and spread of the disease is poorly controlled. As the international trade markets are the major source of Salmonella, blood contaminated feeds and poor sanitization may lead to its major spread.

\section{Salmonella in Pakistan}

Poultry sector is the second largest industry of economic importance in Pakistan. It plays a major role in the national gross domestic product (Hussain et al. 2015). In Pakistan, the total population of poultry birds is approximately 1105.91 million. Salmonella infections are comparatively more common in poultry and have great public health importance due to consumption of poultry products that may be contaminated with these pathogens (Vandeplas et al. 2010). Rearing of backyard poultry is an emerging interest of people around the country (Ali et al. 2018; Achakzai et al. 2020; Ahmed et al. 2021). This trend might possibly increase Salmonella outbreaks linked to these birds. For Salmonella, commercial chicken flocks reared for meat and eggs are usually monitored in Pakistan (Soomro et al. 2011; Shahzad et al. 2012; Uddin et al. 2018; Wajid et al. 2018), but no quantitative epidemiological investigations have been carried out to calculate prevalence of various Salmonella species in backyard chickens. Also, there is inadequate data available to assess the associated risk factors with this infection (Khan et al. 2012). Studies in other developing countries, like Iran (Khaltabadi et al. 2019), India (Balakrishnan et al. 2018) and Paraguay (Huarcaya Ramírez 2020) have suggested great load of Salmonella in chickens and other species.

Health authorities in Pakistan have gathered reports which provide a threat about an ongoing outbreak of extensively drug resistant (XDR) typhoid fever caused by Salmonella. It started to emerge in the Hyderabad district of Sindh province during November 2016 (Khan 2019; Qamar et al. 202ob). An increasing trend of typhoid fever cases caused by antimicrobial resistant strains of $\mathrm{S}$. enterica serovar typhi ( $S$. typhi) poses a notable public health concern in the region.
From January to December 2018, numerous samples from suspected cases of Salmonellosis were examined and Salmonella typhi was found to be prevalent in Karachi; moreover, it was an impending threat of extensive drug resistance (Hussain et al. 2019). It has been established through a number of studies that antimicrobial resistance to ciprofloxacin by both Salmonella typhi and Salmonella paratyphi has been found among the populations in the country (Table 1 ).

\section{Taxonomic Classification}

These bacteria are motile in nature and are mainly concerned with the animals (Ashton et al. 2016; Ryan et al. 2017). This genus has only species named as Salmonella bongori and $S$. enterica. The subspecies of S. enterica is mostly detected from the infections of humans and animals (Löfström et al. 2015; Ullah et al. 2017; Chiller 2019; Haley et al. 2019). The genus Salmonella nearly contains 2,60o serotypes, which are formed on the basis of their flagellar $(\mathrm{H})$ and somatic (O) antigens. S. typhimurium and $\mathrm{S}$. enteritidis are the most common serotypes related to the humans, but other serotypes can also cause infection.Among these serotypes, $S$. newport, $S$. virchow and $S$. infantis are most important ones (Fandiño and Verjan 2019).

Typhoid fever in humans is caused by the serotypes $S$. paratyphi and $S$. typhi, which are the most severe form of Salmonella infection, but most common infections are related to the non-typhoid infections, which normally cause gastroenteritis. It is characterized by symptoms like diarrhea, abdominal cramps, emesis and nausea, which normally last for one to seven days. In healthy adult individuals, the symptoms of the infection are less severe and only cause less than $1 \%$ mortality due to septicemia (Katz et al. 2019). In weak individuals, a dose range from 10-10o cells can initiate infection. The chances of infection are also higher when the food having high fat, like cheese and chocolate, is contaminated.

\section{Clinical Aspects}

Salmonella infection may manifest either as a self-limiting diarrhea/gastroenteritis that is related to non-typhoidal Salmonella (NTS), or as typhoidal fever that involves septicemia and chronic diarrhea and can be life threatening (Barrow and Methner 2013; Cox and Pavic 2014). The first form, NTS, is usually linked with inflammatory diarrhea, vomiting, nausea and abdominal pain (Smith et al. 2016). The severity of infection, like many other infectious diseases, depends upon host immune status, genetic makeup, the specie involved, serovar and dose of inoculation of the pathogen. In typhoidal fever, the microorganisms can spread to the circulatory system, attacking the lymphoid tissues of the intestinal tract.

On the off chance that the circulatory system is engaged with the disease, at that point it can affect any organ, including the bones, gallbladder, liver and meninges, yet its odds of event is under $5 \%$. About 12-72 hours is the 
Table 1: Distribution of reported XDR typhoid fever cases in Sindh province, Pakistan [1 November 2016 through 9 December 2018] Source: WHO (2021)

\begin{tabular}{lcccc}
\hline Year & \multicolumn{3}{c}{ Districts in Sindh province } & \multirow{2}{*}{ Total } \\
\cline { 2 - 4 } & Karachi & Hyderabad & Other districts & \\
\hline 2016 & 0 & 11 & 0 & 11 \\
2017 & 175 & 488 & 67 & 730 \\
2018 & 3483 & 906 & 144 & 4533 \\
Total & 3658 & 1405 & 211 & 5274 \\
\hline
\end{tabular}

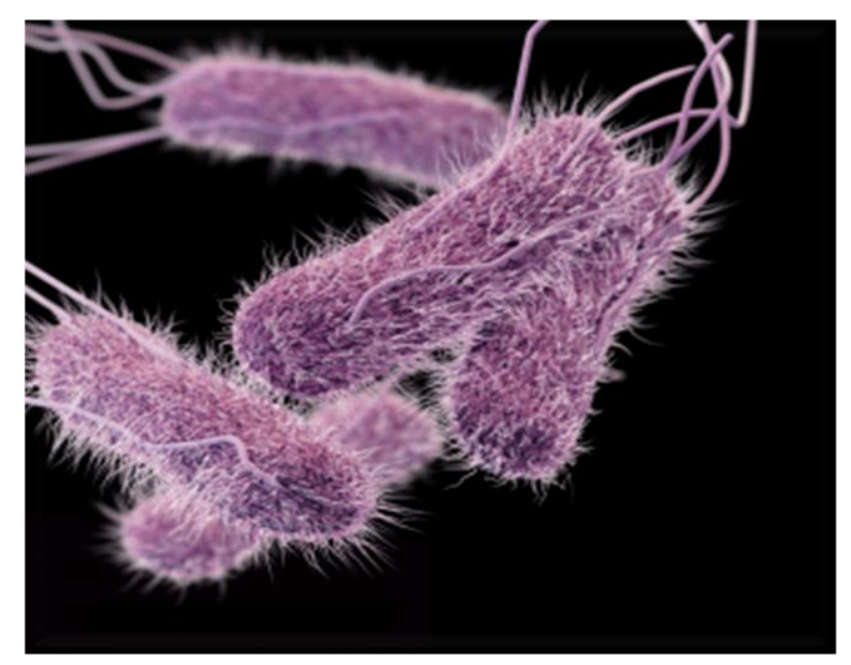

Fig. 1: $\mathrm{A}_{3} \mathrm{D}$ view of Salmonella serotype typhi (Source, CDC).

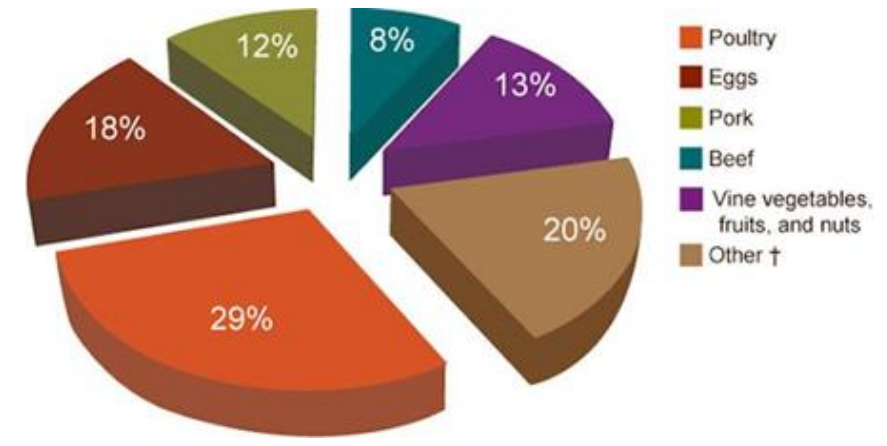

Fig. 2: Diffèrent sources of Salmonella transmissions in human population (Source : CDC website)

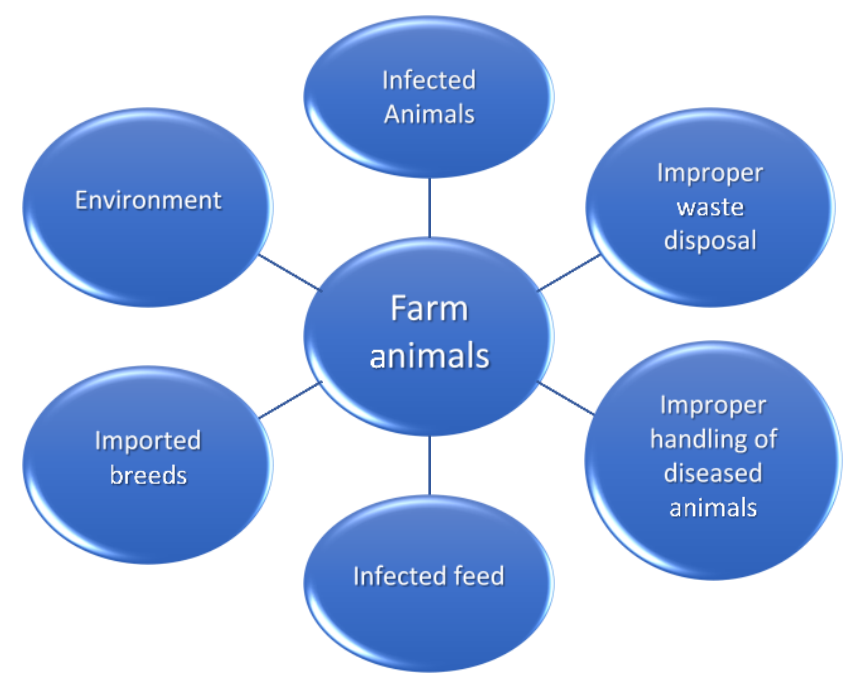

Fig. 3: Schematic illustration of Salmonella spp. indicating the source of infection and transmission modes. brooding time frame for the Salmonellosis contaminations (Khan et al. 2019). The high hazard bunches for creating disease are kids under five years of age, aged persons and the individuals who are immunocompromised. The seriousness of contamination, in the same way as for other irresistible illnesses, relies on insusceptible status, hereditary cosmetics, the specie in question, serovar and status of vaccination against the pathogen (Asmar and Abdel-Haq 2016).

Although salmonellosis is seen in most domestic animals, pregnant and lactating young mammals and birds are found to be most susceptible (Wisittipanit et al. 2020). Cattle can be chronically infected, and therefore, may serve as carriers within the herd without showing any clinical signs. It has been reported that a single carrier cow can shed one billion Salmonellae per day through the feces (Gast and Porter Jr 2020). Abortion is the most common clinical consequence associated with Salmonella dublin (Sánchez-Miguel et al. 2018). Salmonellosis in cattle may cause watery or bloody diarrhea. The other symptoms include fever, depression, anorexia, dehydration, and endotoxemia. In rare cases, the infected cattle may suffer from respiratory disease and abortion, which is associated with high mortality rates (Hadimli et al. 2017).

\section{Sources and Transmission}

In poultry, Salmonella infections are transmitted horizontally, as well as vertically. They can also spread by contamination of the environment like soil, quilt, boxes used for nests, eggs, drinkers and waterers. Mechanical transmission occurs through the insects, rodents, wild birds, equipment, vehicles, clothing, unwashed hands, etc. (Chousalkar and Gole 2016). The infected animals, especially poultry and pigs, often do not show signs of disease (Nirmala et al. 2018) and are therefore, a major cause of disseminating the infection to other herd members and possibly to other humans and animals. According to CDC, poultry is the most common single food source associated with Salmonellosis in humans (Fig. 2).

According to Hale et al. (2012), about eleven percent (11\%) Salmonella infections reported in humans are principally arise from animal handling. At the farm level, infection may be acquired by failure of adaptation of proper biosecurity measures. Introduction of new infected animals in the herd, unhygienic water and feed sources and improper handling of diseased animals may lead to transmission of Salmonella infection (Fig. 3).

\section{Diagnostic Approaches}

Conventionally, Salmonella spp. can be isolated first through non-selective pre-enrichment phase and after that selective enrichment is done, which is then coated on selective agars and alleged colonies are examined serologically and biochemically (Lee et al. 2015). Many regulatory agencies of USDA, like International Organization for Standardization, Association of Official 
Analytical Chemists (AOAC), Food Safety and Inspection Service and Food and Drug Administration (FDA) have standardized many methods for the enrichment of Salmonella. Currently, the international standard operation (ISO) horizontal method validates buffered peptone water to be used as pre-enrichment media and after that Rappaport-Vassiliadis (soya base) and MullerKauffmann Tetrathionate-Novobiocin (MKTTn) selective enrichments are used (ISO 2007). Many other regulatory agencies also suggest standard methods for the isolation of Salmonella, which are fundamentally similar to ISO 6579:2002.

Instant Salmonella detection methods include molecular cloning and recombinant DNA technology. These quick methods allow the recognition of Salmonella spp. in samples and provide reliable results within a few hours to a day (Ferretti et al. 2001; Alakomi and Saarela 2009). New selective media, modified or improved conventional procedures, immunology-based and nucleic acid-based assays are commercially available rapid methods for the detection of Salmonella (Iqbal et al. 2000; Alakomi and Saarela 2009; Eijkelkamp et al. 2009). ELISA and PCR are more sensitive and specific as compared to these conventional methods (Lee et al. 2015). ELISA and PCR, after undergoing enrichment, can detect the concentration of Salmonella at the sensitivity level of 104$105 \mathrm{ml}^{-1}$ and $104 \mathrm{ml}^{-1}$, respectively. Circumstantial microflora, presence of non-culturable cells, sample medium and inhibitory constituents (like adipose tissue, polysaccharides, proteins, hefty metals, organic composites and antibiotics) affect the sensitivity and specificity of these procedures (Naravaneni and Jamil 2005; Mozola 2006; Alakomi and Saarela 2009).

With the introduction of molecular techniques, the diagnosis of bacterial species like Salmonella has been revolutionized. The diverse serotypes of Salmonella can be serotyped accurately, using these state-of-the-art molecular tools.

\section{Recent Outbreaks around the Globe}

Outbreaks of typhoid fever have been controlled to some extent in developed countries, but illness caused by NTS still prevails both in developed and developing countries across the globe. Most of the outbreaks in humans are associated with consumption of contaminated food from animal origin. In Germany, a study conducted by Robert Koch institute has shown incidence of different Salmonella serovars during 2004-2010. According to WHO, there was an outbreak of Extensively DrugResistant (XDR) typhoid fever reported from Sindh province, Pakistan, which caused a total of 5707 cases (Chatham-Stephens et al. 2019).

A total of 53 Salmonella outbreaks related with live poultry have been recorded in United States between 1990 and 2014. These outbreaks caused around 2630 illnesses, 387 hospitalizations, and 5 deaths (Basler et al. 2016). Between September-October 2005, three different outbreaks of Salmonellosis were reported from Kerala, India poultry farms (Rajagopal and Mini 2013).
In New Hampshire, an outbreak of S. typhimurium in humans was reported in 2013. The outbreak was related to chicken jerky pet treats (Cavallo et al. 2015).

On October 17, 2018, a multistate outbreak of Salmonella infantis, related to under cooked chicken products, was reported to the Center for Disease Control and Prevention (CDC). In that outbreak, ninety-two cases were reported from twenty-nine states of United States. Twenty-one people were hospitalized, and no deaths were reported. Monitoring of the outbreak was done by the United States Department of Agriculture (USDA).

In July 19, 2017, different commercial, scientific, college and university coaching laboratories of Microbiology showed outbreak of S. typhimurium, involving the different states. From 16 states of US, 24 individuals were reported with infection. Hospitalized number of individuals was 6 , and no mortality was recorded (CDC, 2018).

On October 6, 2016 CDC and the US Department of Agriculture studied 8 outbreaks of human Salmonella disease related to exposure with live poultry in backyard congregates. From 48 states, 8 outbreaks were reported, infecting 895 individuals with Salmonella strains (Control and Prevention 2014).

\section{Worldwide Surveillance of Salmonella}

This is critically important that detection methods should be consistent and integrated for the surveillance of Salmonella to improve the food safety standards (Ghafir et al. 2005). It is vital to run proper surveillance for the whole food chain by checking the feed and ingredients of feed for contamination of Salmonella (Alakomi and Saarela 2009; Mead et al. 2010). To efficiently avert and control the Salmonella infections, regulation, standardization, and worldwide surveillance programs are also compulsory. Information about the sampling technique, their storage and other important steps in the analysis of Salmonella is present in the Nordic Committee of Food Analysis (Validation 2002; Feldsine et al. 2003; ISO 2007; Löfström et al. 2010). In the form of EU Zoonosis Monitoring Directive, Regulation Number 2160/2003, and FDA Food Code regulations and guidelines are present in many countries (Commission 2003a\&b; Food and Adminstration 2008). To decrease pathogens of Salmonella in the different stages of feed and its production, these guidelines and regulations provide the required information and control methods. They also help to develop the regulatory policies and the food safety rules (Kadykalo et al. 2018; Firestone 2020).

\section{Pathogenesis}

Salmonella displays momentous properties when it attacks non-phagocytic human host cells, adequately instigating its phagocytosis to enter the host cell. The phenomenal hereditary qualities behind this cunning procedure can be found in the Salmonella pathogenicity islands (SPI), in the quality bunches in the huge locale of chromosomal DNA and in the coding structures 
associated with the intrusion cycle (Lenchenko et al. 2019). At the point when microscopic organisms enter the stomach related lot from defiled water or food, they will in general attack the epithelial cells that line the intestinal divider.

The seriousness of Salmonella diseases in people relies upon the serotype of the organism and the soundness of the human host. Youngsters under 5 yearsof age, the aged and immunocompromised patients are more susceptible to Salmonella contaminations than healthy individuals. Practically, all Salmonella strains are pathogenic, as they can attack human host cells, repeat and endure, prompting a conceivable sickness (Smith et al. 2016; Dougnon et al. 2017; Garedew et al. 2018). Illness usually lasts for 4-7 days. In typhoidal fever, the bacteria can spread to the bloodstream, invading the lymphoid tissues of the intestinal tract (Barrow and Methner 2013; Cox and Pavic 2014). If the bloodstream is involved in the infection, then it can affect any organ, including the bones, gallbladder, liver and meninges, but its chances of occurrence is less than $5 \%$. Almost, 12-72 hours is the incubation period for the Salmonella infections (Khan et al. 2019). The high-risk groups for developing infection are children less than five years of age, elderly and persons who are immunocompromised.

The complex pathogenesis of systemic $S$. enterica infections correlates with the presence of a large number of defensive, as well as offensive, virulence factors (Groisman and Ochman 1997), with more than 300 genes have been identified as regulatory genes in Salmonella (Yoon et al. 2011). Salmonella has pathogenicity island SPIs, responsible for effects like adhesion, toxicity and invasion. There are 14 types of virulence regulatory genes, including SpvR, FruR, IHF, PhoP/PhoQ, SsrA/SsrB, SlyA, Hnr, RpoE, SmpB, CsrA, RpoS, CRP, OmpR/EnvZ, and Hfq (Yoon et al. 2009). These are often associated with transfer RNA (tRNA) and mobile genetic elements.

\section{Control and Prevention}

Livestock and poultry are the main reservoir hosts to cause Salmonellosis in humans, so humans exposure can be considerably reduced by decreasing infectious pathogens of Salmonella present in these animals (Arya et al. 2017). To prevent transmission of the disease, all feeds of animals are cured before their dissemination to kill the bacteria. Animals detected positive for Salmonella, should be reported and animals related to food safety should also be regularly examined for Salmonella (Milho et al. 2018). To prevent the Salmonella enteritidis infection in flocks, these strategies are adopted by the egg producers in the US as a control measure. Routine disinfection in and around the poultry farms should be done. Pets and rodents should also be controlled properly (Ribas et al. 2016), and feed should be obtained from the benign foundations. There should be routine investigation for the Salmonella enteritidis in chickens. Pasteurization of the eggs laid by the diseased egg-laying chickens should be done, and the infected birds should be removed.

Intricate food safety insurances are necessary for the control of Salmonellosis. This can be implemented by the prevention of ready-to-eat foods during their processing and refrigerated foods should also be sufficiently protected by controlling the survival and growth of Salmonella. All the persons dealing with the raw and cooked food and utensils should be cleaned with water and soap, and different food items such as meat, milk and vegetables are advised to be cooked at designated temperature to kill all the microbes. We should introduce the culture of germ-free slaughtering of animals, consumption of pasteurized milk, and completely cooked items to decrease the risk of being infected (Bao et al. 2015). Many serovars of Salmonella are extra resilient to antibiotics (das Neves et al. 2016). When antibiotics are inevitably required, then some of these can be used in humans for treatment and control of infections. For the prevention of this causative agent, judicial use of antibiotics among the humans and animals is imperious (Shrestha et al. 2016). Surveillance programs and subtyping of isolates are important for the control of Salmonella. By investigating outcomes of Salmonella subtyping from animals used for food, ecological illustrations, and humans, public health administrators can lure deductions about foundations of human disease and emphasis on control strategies consequently.

Immunization against Salmonella Species of Public Health Significance

To lessen the Salmonella infections, vaccination of chickens is necessary for Salmonella and other interference infections. Two licensed vaccines are largely available against Salmonella (Gayet et al. 2017), but till now no one has been executed at the country level. These vaccines are live attenuated,Ty21a (Fraser et al. 2007) and Vi capsular polysaccharide (Gayet et al. 2017). They cause lower immunogenicity in younger children and, therefore, the use of these vaccines is reduced in the highrisk individuals and no one is authorized to use in children of less than two years of age. The drawback of these vaccines is that they are directed against $S$. typhimurium, with no cross immunity for other three invasive types of serovars of $S$. enterica, paratyphi A, typhimurium and enteritidis.

In Pakistan, typhoid conjugate vaccine (Typbar TCV) is largely practiced and a research team is working on mass vaccination against the outbreak to target a local town, and quite healthy response is achieved (Qamar et al. 2020a). Pakistan became the pioneer state and the first country to introduce the WHO recommended typhoid conjugate vaccine into routine vaccination programs and this vaccine has high efficacy, with added benefit of longlasting immunity. The vaccine is now being administered to the individuals aged 6 months or older since 2019, with fewer adverse effects (Qamar et al 202ob).

\section{Conclusion}

Salmonellosis is an important zoonotic disease of animal origin; poultry meat, eggs and their products are the 
common sources of transmission to humans. Salmonellosis has posed a serious threat to the public health (Zdragas et al. 2012), as it is thought to be the most frequent food borne pathogen worldwide. During the period of $1983-87$ in US, one third outbreaks of human Salmonellosis were linked with poultry chicken and eggs (Tauxe 1991). In Pakistan, poultry chicken is the main type of meat being consumed, as in many other countries. Apart from commercial poultry farming, backyard poultry is also reared in urban and rural areas to meet the nutritional requirements and they are also vulnerable to Salmonella infection (Bhuvaneswari et al. 2015). Salmonella has the ability to attach with any part of gastrointestinal tract in the absence of other microflora, where it can colonize and multiply. It can also be disseminated to the environment, humans and other animals via feces (AL-Kubaisi et al. 2020; Santana et al. 2020). Therefore, fecal swabs or samples are used as true representative to confirm the presence of Salmonella in the intestinal wall of poultry chicks (El-Fakar and Rabie 2009).

Irrational use of antibiotics in poultry and livestock farming has resulted in the rise of antibiotic resistance among food producing animals, which envisage a major threat to production industry. The rise of MDR Salmonella causes an escalating risk for health of human population. Out of the 10 serotypes that CDC has commonly reported from human infections in the US, 8 comprise at least a few isolates that showed resistance to five or more antimicrobic drugs (Varma et al. 2005). MDR $S$. typhimurium isolates commonly exist in two resistance forms including, (i) resistance to ampicillin, streptomycin, kanamycin, tetracycline and sulfamethoxazole or, (ii) resistance to ampicillin, chloramphenicol, streptomycin, sulfamethoxazole, and tetracycline, the resistance type is typically associated with S. typhimurium DT104 (Soler et al. 2006). Regarding antibiotic resistance, production of extended spectrum Beta Lactamase invokes a major threat to livestock and poultry production system. This threat not only affects animals, but also humans, which are at risk of being infected in direct food chain (Witte 1998). A current study appraises the presence of extended spectrum Beta lactamase in gut $S$. typhimurium in poultry food chain. Double Disc screening test was employed to differentiate ESBL producing organism from non ESBL producing $S$. typhimurium (Nadimpalli et al. 2019).

Hence, proper legislation holds the key to lessen the risk of antimicrobial resistance among animals and human populations. The principal factor for inhibiting the spread of antimicrobial resistance throughout the food chain is identical to the non-resistant foodborne pathogens that may include the appropriate food management, and food formulation methods. Additionally, there is substantial proof that the use of antimicrobials for the treatment of food animals, their growth promotion and prophylaxis, increase the prevalence of resistance in human pathogens (Singer et al. 2007; Saharan et al. 2020).

Immunization against different Salmonella serovars in human population is the ultimate solution for public health challenge. Pakistan, being the first country in the region following typhoid conjugate vaccine, has permitted the use of vaccine for the individuals, even above 6 months of age.

\section{REFERENCES}

Achakzai KB et al., 2020. Backyard chicken farming role in supplementing household economy of district Quetta, Pakistan. Turkish Journal of Agriculture-Food Science and Technology 8: 568-572.

Ahmed T et al., 2021. Pakistan's backyard poultry farming initiative: Impact analysis from a public health perspective. Tropical Animal Health and Production 53: 1-12.

AL-Kubaisi S et al., 2020. Isolation and identification of facultative anaerobic bacteria from feces of pet dogs. Medico Legal Update 20: 783-787.

Alakomi HL and Saarela M, 2009. Salmonella importance and current status of detection and surveillance methods. Quality Assurance and Safety of Crops and Foods 3: 142-152.

Ali $\mathrm{M}$ et al., 2018. Prevalence and phylogenetics of $\mathrm{H}_{9} \mathrm{~N}_{2}$ in backyard and commercial poultry in Pakistan. Avian Diseases 62: 416-424.

Archambault $\mathrm{M}$ et al., 2006. Molecular characterization and occurrence of extended-spectrum $\beta$-lactamase resistance genes among Salmonella enterica serovar Corvallis from Thailand, Bulgaria and Denmark. Microbial Drug Resistance 12: 192-198.

Arshad MM et al., 2008. Epidemiologic attributes of invasive non-typhoidal Salmonella infections in Michigan, 1995-2001. International Journal of Infectious Diseases 12: 176-182.

Arya G et al., 2017. Epidemiology, pathogenesis, genoserotyping, antimicrobial resistance, and prevention and control of non-typhoidal Salmonella serovars. Current Clinical Microbiology Reports 4: 4353 .

Ashton PM et al., 2016. Identification of Salmonella for public health surveillance using whole genome sequencing. Peer Journal 4: e1752.

Asmar BI and Abdel-Haq N, 2016. Nontyphoidal Salmonella infection in children: Relation to bacteremia, age and infecting serotype. Infectious Diseases 48: 147-151.

Balakrishnan S et al., 2018. Prevalence of Salmonella in chicken meat and its slaughtering place from local markets in Orathanadu, Thanjavur district, Tamil Nadu. Journal of Entomology and Zoology Studies 6: 2468-2471.

Bao $\mathrm{H}$ et al., 2015. Bio-control of Salmonella enteritidis in foods using bacteriophages. Viruses 7: 4836-4853.

Barrow PA and Methner U, 2013. Salmonella in domestic animals. ePub 9781789244496 CABI.

Basler C et al., 2016. Outbreaks of human Salmonella infections associated with live poultry, United States, 1990-2014. Emerging Infectious Diseases 22: 1705.

Bhuvaneswari $\mathrm{M}$ et al., 2015. Prevalence of multidrugresistant (MDR) Salmonella enteritidis in poultry and 
backyard chicken from Tiruchirappalli. Indian Microbiology Journal 5: 28-35.

Birhanu BT et al., 2018. Inhibition of Salmonella typhimurium adhesion, invasion, and intracellular survival via treatment with methyl gallate alone and in combination with marbofloxacin. Veterinary Research 49: 1-11.

Bogomazova AN et al., 2020. Mega-plasmid found worldwide confers multiple antimicrobial resistance in Salmonella infantis of broiler origin in Russia. International Journal of Food Microbiology 319: 108497.

Cavallo SJ et al., 2015. Human outbreak of Salmonella typhimurium associated with exposure to locally made chicken jerky pet treats, New Hampshire, 2013. Foodborne Pathogens and Disease 12: 441-446.

Chatham-Stephens $\mathrm{K}$ et al., 2019. Emergence of extensively drug-resistant Salmonella typhi infections among travelers to or from Pakistan-United States, 2016-2018. Morbidity and Mortality Weekly Report 68: 11.

Chiller TM, 2019. Salmonella/foodborne outbreaks in USA. Pathology 51: S59-S6o.

Chousalkar K and Gole VC, 2016. Salmonellosis acquired from poultry. Current Opinion in Infectious Diseases 29: 514-519.

Cocciolo G et al., 2020. Evidence of vector borne transmission of Salmonella enterica enterica serovar Gallinarum and fowl typhoid disease mediated by the poultry red mite, Dermanyssus gallinae (De Geer, 1778). Parasites and Vectors 13: 1-10.

Commission E, 2003a. Directive 2003/99/EC of the European Parliament and of the Council of 17 November 2003 on the monitoring of zoonoses and zoonotic agents, amending Council Decision 90/424/EEC and repealing Council Directive 92/117/EEC. Official Journal of European Union 50: 3140.

Commission E, 2003b. Regulation (EC) No. 2160/2003 of the European Parliament and of the Council of 17 November 2003 on the control of salmonella and other specified food-borne zoonotic agents. Official Journal of European Union 103: 86-93.

Control and Prevention, 2014. Eight multistate outbreaks of human Salmonella infections linked to small turtles (final update). CDC Website: October 18, 2013 3:0o PM ET.

Cox J and Pavic A, 2010. Advances in enteropathogen control in poultry production. Iournal of Applied Microbiology 108: 745-755.

Crouch CF et al., 2020. Reduction in intestinal colonization and invasion of internal organs after challenge by homologous and heterologous serovars of Salmonella enterica following vaccination of chickens with a novel trivalent inactivated Salmonella vaccine. Avian Pathology 49: 666-677.

Dagnew B et al., 2020. Prevalence and antimicrobial susceptibility of Salmonella in poultry farms and incontact humans in Adama and Modjo towns, Ethiopia. Microbiology Open 9: e1067.
Dantas ST et al., 2018. Cross-contamination and biofilm formation by Salmonella enterica serovar Enteritidis on various cutting boards. Foodborne Pathogens and Disease 15: 81-85.

Das Neves GB et al., 2016. Salmonella heidelberg isolated from poultry shows a novel resistance profile. Acta Scientiae Veterinariae 44: 1-6.

Das Q et al., 2019. Transcriptional profiling of Salmonella enterica serovar Enteritidis exposed to ethanolic extract of organic cranberry pomace. Plos One 14: eo219163.

Deng $W$ et al., 2020. Heavy metals, antibiotics and nutrients affect the bacterial community and resistance genes in chicken manure composting and fertilized soil. Journal of Environmental Management 257: 109980.

Dougnon T et al., 2017. Traditional treatment of human and animal Salmonelloses in Southern Benin: Knowledge of farmers and traditherapists. Veterinary World 10: 580 .

Eguale $\mathrm{T}$ et al., 2017. Genetic markers associated with resistance to beta-lactam and quinolone antimicrobials in non-typhoidal Salmonella isolates from humans and animals in central Ethiopia. Antimicrobial Resistance and Infection Control 6: 110.

Eijkelkamp J et al., 2009. Suitability of rapid detection methods for Salmonella in poultry slaughterhouses. Food Analytical Methods 2: 1-13.

Eikmeier D et al., 2018. Incubation period for outbreakassociated, non-typhoidal Salmonellosis cases, Minnesota, 2000-2015. Epidemiology and Infection 146: 423-429.

El-Fakar SAZ and Rabie NS, 2009. Immunogenic properties of outer membrane proteins of Salmonella in chicken. Global Veterinaria 3: 75-79.

Eng S-K et al., 2015. Salmonella: A review on pathogenesis, epidemiology and antibiotic resistance. Frontiers in Life Science 8: 284-293.

Fandiño LC and Verjan N, 2019. A common Salmonella enteritidis sequence type from poultry and human gastroenteritis in Ibagué, Colombia. Biomédica 39.

Feldsine PT et al., 2003. Detection of Salmonella in fresh cheese, poultry products and dried egg products by the ISO 6579 Salmonella culture procedure and the AOAC official method: Collaborative study. Journal of AOAC International 86: 275-295.

Ferretti R et al., 2001. Twelve-hour PCR-based method for detection of Salmonella spp. in food. Applied Environmental Microbiology 67: 977-978.

Firestone M, 2020. Restaurants and Salmonella: Using surveillance data to improve policy development for the enhancement of food safety. Thesis: University of Minnesota, USA: https://hdl.handle.net/11299/215155.

Food K and Adminstration D, 20o8. Food code 999: 542.

Fraser A et al., 2007. Typhoid fever vaccines: Systematic review and meta-analysis of randomised controlled trials. Vaccine 25: 7848-7857.

Garedew L et al., 2018. Diagnosis and treatment of human Salmonellosis in Addis Ababa City, Ethiopia. 
BioMed Research International 2018: ID 6406405, https://doi.org/10.1155/2018/6406405

Gast RK and Porter Jr RE, 2020. Salmonella infections. Diseases of Poultry 2020: 717-753.

Gayet R et al., 2017. Vaccination against Salmonella infection: The mucosal way. Microbiology and Molecular Biology Reviews 81: eoooo7-17.

Gerner-Smidt P et al., 2006. PulseNet USA: A five-year update. Foodborne Pathogens and Disease 3: 9-19.

Ghafir Y et al., 2005. Belgian surveillance plans to assess changes in Salmonella prevalence in meat at different production stages. Journal of Food Protection 68: 2269-2277.

Groisman EA and Ochman Tim HJ, 1997. How Salmonella became a pathogen. Trends in Microbiology 5:343-349.

Hadimli HH et al., 2017. Serotypes of Salmonella isolated from feces of cattle, buffalo, and camel and sensitivities to antibiotics in Turkey. Turkish Journal of Veterinary and Animal Sciences 41: 193-198.

Hale CR et al., 2012. Estimates of enteric illness attributable to contact with animals and their environments in the United States. Clinical Infectious Diseases 54: 472-479.

Haley BJ et al., 2019. Complete genome sequence of a Salmonella enterica subsp. enterica serovar fresno isolate recovered from a bovine lymph node. Microbiology Resource Announcement 8: eo1338-18.

Hassan R et al., 2018. Multistate outbreak of Salmonella paratyphi B variant L (+) tartrate $(+)$ and Salmonella Weltevreden infections linked to imported frozen raw tuna: USA, March-July 2015. Epidemiology and Infection 146: 1461-1467.

Hendriksen RS et al., 2011. Global monitoring of Salmonella serovar distribution from the World Health Organization Global Foodborne Infections Network Country Data Bank: results of quality assured laboratories from 2001 to 2007. Foodborne Pathogens and Disease 8: 887-90o.

Huarcaya Ramírez FR, 2020. Serotipificación y detección genética de Salmonella spp. De origen aviar. Tesis EP Medicina Veterinaria 547.

Hurley D et al., 2014. Salmonella-host interactionsmodulation of the host innate immune system. Frontiers in Immunology 5: 481.

Hussain A et al., 2019. Typhoidal Salmonella strains in Pakistan: An impending threat of extensively drugresistant Salmonella typhi. European Journal of Clinical Microbiology and Infectious Diseases 38: 2145-2149.

Hussain J et al., 2015. An overview of poultry industry in Pakistan. World's Poultry Science Journal 71: 689-70o.

Iqbal SS et al., 2000. A review of molecular recognition technologies for detection of biological threat agents. Biosensors and Bioelectronics 15: 549-578.

ISO, 2007. Microbiology of food and animal feeding stuffs. Horizontal method for the detection of Salmonella spp. Norm ISO 6579: 2002/Amd 1: 2007. In: International organization for standardization publication geneva, Switzerland.
Issenhuth-Jeanjean S et al., 2014. Supplement 2008-2010 to the White-Kauffmann-Le Minor scheme. Research in Microbiology 165: 526-530.

Jafari R et al., 2007. An investigation into Salmonella infection status in backyard chickens in Iran. International Journal of Poultry Science 6: 227-229.

Jantsch J et al., 2011. Cellular aspects of immunity to intracellular Salmonella enterica. Immunological Reviews 240: 185-195.

Johansson MH et al., 2021. Detection of mobile genetic elements associated with antibiotic resistance in Salmonella enterica using a newly developed web tool: Mobile Element Finder. Journal of Antimicrobial Chemotherapy 76: 101-109.

Kaczorek-Łukowska E et al., 2020. Can domestic pigeon be a potential carrier of zoonotic Salmonella? Transboundary and Emerging Diseases 68: 2321-2333.

Kadykalo SV et al., 2018. Passive surveillance of antimicrobial resistance in Salmonella and Escherichia coli isolates from Ontario livestock, 20072015. Canadian Veterinary Journal 59: 617.

Katz D et al., 2019. Correlates of non-typhoidal Salmonella bacteraemia: A case-control study. International Journal of Infectious Diseases 81: 170175.

Kaufmann $\mathrm{SH}$ et al., 20o1. Introduction: microbiology and immunology: Lessons learned from Salmonella. Microbes and Infection 3: 1177-1181.

Khaltabadi RF et al., 2019. Salmonella typhimurium in Iran: Contribution of molecular and IS 200 PCR methods in variants detection. Plos One 14: eo213726.

Khan E, 2019. Drug resistant typhoid fever: An emerging public health crisis. Rawalpindi Medical Journal 44: 13.

Khan MI et al., 2012. Risk factors associated with typhoid fever in children aged 2-16 years in Karachi, Pakistan. Epidemiology and Infection 140: 665-672.

Khan SB et al., 2019. Phentotypic, gentotypic antimicrobial resistance and pathogenicity of Salmonella enterica serovars Typimurium and enteriditis in poultry and poultry products. Microbial Pathogenesis 129: 118-124.

Lambertini E et al., 2016. Transmission of bacterial zoonotic pathogens between pets and humans: The role of pet food. Critical Reviews in Food Science and Nutrition 56: 364-418.

Lee KM et al., 2015. Review of Salmonella detection and identification methods: Aspects of rapid emergency response and food safety. Food Control 47: 264-276.

Lenchenko E et al., 2019. Aspects of Salmonellosis pathogenesis using chicken models. Balian Medicine Journal 8: 206-210.

Lima $\mathrm{T}$ et al., 2019. Plasmid-mediated colistin resistance in Salmonella enterica: A review. Microorganisms 7: 55 .

Löfström $C$ et al., 2010. Validation of a 20-h real-time PCR method for screening of Salmonella in poultry faecal samples. Veterinary Microbiology 144: 511-514.

Löfström C et al., 2015. Salmonella: Salmonellosis. In: Encyclopedia of Food and Health. Academic Press 
Majowicz SE et al., 2010. The global burden of nontyphoidal Salmonella gastroenteritis. Clinical Infectious Diseases 50: 882-889.

Manning J et al., 2015. Screening for Salmonella in backyard chickens. Preventive Veterinary Medicine 120: 241-245.

Mead G et al., 2010. Scientific and technical factors affecting the setting of Salmonella criteria for raw poultry: A global perspective. Journal of Food Protection 73: 1566-1590.

Michael GB et al., 2005. Class 1 integron-associated gene cassettes in Salmonella enterica subsp. enterica serovar Agona isolated from pig carcasses in Brazil. Journal of Antimicrobial Chemotherapy 55: 776-779.

Milho $C$ et al., 2018. Control of Salmonella enteritidis on food contact surfaces with bacteriophage PVP-SE2. Biofouling 34: 753-768.

Monte DF et al., 2019. Genomic features of high-priority Salmonella enterica serovars circulating in the food production chain, Brazil, 2000-2016. Scientific Reports 9: 1-12.

Mozola MA, 2006. Genetics-based methods for detection of Salmonella spp. in foods. Journal of AOAC International 89: 517-529.

Muhammad Y et al., 2015. Comparative lethality of Salmonella enteritidis and Salmonella typhimurium in broiler chickens. Indian Journal of Animal Sciences 85: 472-474.

Nadimpalli $M$ et al., 2019. CTX-M-55-type ESBLproducing Salmonella enterica are emerging among retail meats in Phnom Penh, Cambodia. Journal of Antimicrobial Chemotherapy 74: 342-348.

Naravaneni R and Jamil K, 2005. Rapid detection of foodborne pathogens by using molecular techniques. Journal of Medical Microbiology 54: 51-54.

Nascimento GM et al., 2019. Effects of Curcuma longa on the intestinal health of chicks infected with Salmonella typhimurium. Revista Brasileira de Zootecnia 48.

Nguyen TK et al., 2021. Retail fresh vegetables as a potential source of Salmonella infection in the Mekong Delta, Vietnam. International Journal of Food Microbiology 341: 109049.

Nielsen LR, 2009. Overview of pathogenesis, epidemiology and diagnostic tools necessary for successful surveillance and eradication of Salmonella dublin from the Danish cattle population. Prize assignment" Professor Dr. med. hc CO Jensens Mindefond". Department of Large Animal Sciences, University of Copenhagen. 7op.

Nirmala TV et al., 2018. Salmonellosis in Poultry: A Case Report. International Journal of Current Microbiology and Applied Sciences 7: 2347-2349.

Pal $\mathrm{M}$ et al., 2020. Animals and food of animal origin as a potential source of Salmonellosis: A review of the epidemiology, laboratory diagnosis, economic impact and public health significance. American Journal of Microbiological Research 8: 48-56.

Park B et al., 2017. Classification of Salmonella serotypes with hyperspectral microscope imagery. Annals of
Clinical Pathology 5: 1108.

Qamar FN et al., 2020a. Strategies to improve coverage of typhoid conjugate vaccine (TCV) immunization campaign in Karachi, Pakistan. Vaccine 8: 697.

Qamar FN et al., 202ob. Adverse events following immunization with typhoid conjugate vaccine in an outbreak setting in Hyderabad, Pakistan. Vaccine 38 : 3518-3523.

Rajagopal R and Mini M, 2013. Outbreaks of Salmonellosis in three different poultry farms of Kerala, India. Asian Pacific Journal of Tropical Biomedicine 3: 496-500.

Rao S et al., 2020. Genomic diversity of class I integrons from antimicrobial resistant strains of Salmonella typhimurium isolated from livestock, poultry and humans. Plos One 15: eo243477.

Raspoet R, 2011. Improving the safety and quality of eggs and egg products. Volume-2: Egg safety and nutritional quality. In: Woodhead Publishing Series in Food Science, Technology and Nutrition 214: 46-61.

Ribas A et al., 2016. Rodents as a source of Salmonella contamination in wet markets in Thailand. VectorBorne and Zzoonotic Diseases 16: 537-540.

Ryan MP et al., 2017. Evaluation of the complex nomenclature of the clinically and veterinary significant pathogen Salmonella. BioMed Research International. Article ID 3782182, 6 pages, 2017.

Saharan VV et al., 2020. Escherichia coli, Salmonella spp., and Staphylococcus aureus susceptibility to antimicrobials of human and veterinary importance in poultry sector of India. Journal of Food Safety 40: e12742.

Sánchez-Miguel C et al., 2018. Sensitivity, specificity and predictive probability values of serum agglutination test titres for the diagnosis of Salmonella dublin culture-positive bovine abortion and stillbirth. Transboundary and Emerging Diseases 65: 676-686.

Sandala JL et al., 2020. A dual-therapy approach for the treatment of biofilm-mediated Salmonella gallbladder carriage. PLoS Pathogens 16: e1009192.

Santana AM et al., 2020. Comparative analysis using pulsed-field gel electrophoresis highlights a potential transmission of Salmonella between asymptomatic buffaloes and pigs in a single farm. Frontiers in Veterinary Science 7: 1-7.

Shahzad A et al., 2012. Prevalence of Salmonella species in hen eggs and egg storing-trays collected from poultry farms and marketing outlets of Faisalabad, Pakistan. Pakistan Journal of Agriculture Sciences 49: 565-568.

Shrestha KL et al., 2016. Re-emergence of the susceptibility of the Salmonella spp. isolated from blood samples to conventional first line antibiotics. Antimicrobial Resistance and Infection Control 5: 22.

Singer S et al., 2007. Workforce perceptions of hospital safety culture: Development and validation of the patient safety climate in healthcare organizations survey. Health Services Research 42: 1999-2021.

Smith SI et al., 2011. Molecular typing of Salmonella spp isolated from food handlers and animals in Nigeria. 
International Journal of Molecular Epidemiology and Genetics 2: 73.

Smith SI et al., 2016. Typhoidal and non-typhoidal Salmonella infections in Africa. European Journal of Clinical Microbiology and Infectious Diseases 35: 19131922.

Soler $\mathrm{P}$ et al., 2006. Antimicrobial resistance in nontyphoidal Salmonella from human sources, Spain, 2001-2003. Journal of Antimicrobial Chemotherapy 58: $310-314$.

Soomro AH et al., 2011. Prevalence and antimicrobial resistance of Salmonella serovars isolated from poultry meat in Hyderabad, Pakistan. Turkish Journal of Veterinary and Animal Sciences 34: 455-46o.

Stear M, 2005. OIE Manual of Diagnostic Tests and Vaccines for Terrestrial Animals (Mammals, Birds and Bees) $5^{\text {th }}$ Edn. Vol. 1 \& 2. World Organization for Animal Health 2004. ISBN 929044622 6.€ 140.

$\mathrm{Su} \mathrm{L}$ and Chiu C, 2007. Salmonella: Clinical importance and evolution of nomenclature. Chang Gung Medical Journal 30: 210.

Tauxe RV, 1991. Salmonella: A postmodern pathogen. Journal of Food Protection 54: 563-568.

Tekintaş $\mathrm{Y}$ et al., 2018. Investigation of antimicrobial susceptibility profile, virulence genes and epidemiological relationship of clinical Salmonella isolates. Short title: Susceptibility and virulence in Salmonella isolates. Turkish Journal of Pharmaceutical Sciences 15: 207-211.

Uddin MN et al., 2018. Antibiotic assays of Salmonella isolated from poultry chicken of various locations in districts Swat. Pure and Applied Biology 7: 78-84.

Ullah $\mathrm{S}$ et al., 2017. Salmonella infection amongst food workers in Lahore. Journal of Ayub Medical College Abbottabad 29: 366.

Validation N, 2002. NV-DOC. Protocol for validation of alternative microbiological methods. In: NordVal Validation Denmark. D-2002-10-22.

Vandeplas S et al., 2010. Salmonella in chicken: Current and developing strategies to reduce contamination at farm level. Journal of Food Protection 73: 774-785.
Varma JK et al., 2005. Antimicrobial-resistant nontyphoidal Salmonella is associated with excess bloodstream infections and hospitalizations. Journal of Infectious Diseases 191: 554-561.

Vohra P et al., 2018. Quantifying the survival of multiple Salmonella enterica serovars in vivo via massively parallel whole-genome sequencing to predict zoonotic risk. Applied Environmental Microbiology 84: e02262-17.

Wajid $M$ et al., 2018. Multiple drug resistance and virulence profiling of Salmonella enterica serovars typhimurium and enteritidis from poultry farms of Faisalabad, Pakistan. Microbial Drug Resistance 25: 133-142.

Wisittipanit $\mathrm{N}$ et al., 2020. CRISPR-2 PCR and high resolution melting profiling for identification and characterization of clinically-relevant Salmonella enterica subsp. enterica. Peer Journal 8: eg113.

Witte W, 1998. Medical consequences of antibiotic use in agriculture. In: American Association for the Advancement of Sciences. Science 279: 996-7.

Wong $\mathrm{V}$ et al., 2018. An extended genotyping framework for Salmonella enterica serovar typhi, the cause of human typhoid. Nature Communication 7: 12827.

Yoon $\mathrm{H}$ et al., 2011. Systems analysis of multiple regulator perturbations allows discovery of virulence factors in Salmonella. BMC Systems Biology 5: 100.

Yoon $\mathrm{H}$ et al., 2009. Coordinated regulation of virulence during systemic infection of Salmonella enterica serovar typhimurium. 5: e1000306.

Younus $\mathrm{M}$ et al., 2011. Food borne disease (Salmonellosis) as public health problem through consuming the meat and eggs of carrier's birds. IJAVMS 5: 111-112.

Younus $\mathrm{M}$ et al., 2009. PCR detection of Salmonella enteritidis and Salmonella typhimurium in poultry feed. Pakistan Journal of Zoology 41: 413-414.

Zdragas A et al., 2012. Prevalence, seasonal occurrence and antimicrobial resistance of Salmonella in poultry retail products in Greece. Letters in Applied Microbiology 55: 308-313. 